\title{
Peroneal Flap: Clinical Application and Cadaveric Study
}

\author{
Yooseok $\mathrm{Ha}^{1}$, Kwan Koo $\mathrm{Yeo}^{2}$, Yibo Piao ${ }^{1,3}$, Sang-Ha Oh ${ }^{1,3}$ \\ ${ }^{1}$ Department of Plastic and Reconstructive Surgery, Chungnam National University, Daejeon; ${ }^{2}$ Jinan Public Health Care Center, Jinan; ${ }^{3}$ Brain \\ Research Institute, Chungnam National University School of Medicine, Daejeon, Korea
}

Background The goal of this study was to investigate the anatomy of the peroneal artery and its perforators, and to report the clinical results of reconstruction with peroneal artery perforator flaps.

Methods The authors dissected 4 cadaver legs and investigated the distribution, course, origin, number, type, and length of the perforators. Peroneal artery perforator flap surgery was performed on 29 patients.

Results We identified 19 perforators in 4 legs. The mean number of perforators was 4.8 per leg, and the mean length was $4.8 \mathrm{~cm}$. Five perforators were found proximally, 9 medially, and 5 distally. We found 12 true septocutaneous perforators and 7 musculocutaneous perforators. Four emerged from the posterior tibia artery, and 15 were from the peroneal artery. The peroneal artery perforator flap was used in 29 patients. Retrograde island peroneal flaps were used in 8 cases, anterograde island peroneal flaps in 5 cases, and free peroneal flaps in 16 cases. The mean age was 59.9 years, and the defect size ranged from $2.0 \mathrm{~cm} \times 4.5 \mathrm{~cm}$ to 8.0 $\mathrm{cm} \times 8.0 \mathrm{~cm}$. All the flaps survived. Five flaps developed partial skin necrosis. In 2 cases, a split-thickness skin graft was performed, and the other 3 cases were treated without any additional procedures.

Conclusions The peroneal artery perforator flap is a good alternative for the reconstruction of soft tissue defects, with a constant and reliable vascular pedicle, thin and pliable skin, and the possibility of creating a composite tissue flap.

Keywords Surgical flaps / Perforator flap / Leg
Correspondence: Sang-Ha Oh Department of Plastic and Reconstructive Surgery, Chungnam National University Hospital, Chungnam National University School of Medicine, 282 Munhwa-ro, Junggu, Daejeon 35015, Korea Tel: +82-42-280-7387

Fax: +82-42-280-7384 E-mail: djplastic@cnu.ac.kr

This research was supported by Basic Science Research Program through the National Research Foundation of Korea (NRF) funded by the Ministry of Science, ICT \& Future Planning (NRF2013R1A1A1057928).

This work was also supported by the research fund of Chungnam National University.

No potential conflict of interest relevant to this article was reported.

Received: 3 Sep $2016 \bullet$ Revised: 27 Dec $2016 \bullet$ Accepted: 4 Jan 2017

pISSN: 2234-6163 • elSSN: 2234-6171 • https://doi.org/10.5999/aps.2017.44.2.136• Arch Plast Surg 2017;44:136-143

\section{INTRODUCTION}

Flap surgery is a fundamental reconstruction method for covering a defect. The recent popularization of microsurgery has rapidly increased the areas to which flap surgery is applicable. Reconstruction using microsurgery, or free flap surgery, is increasingly common, and has become an important area of reconstructive surgery. While these procedures have increased in fre- quency, quality improvement is necessary. To meet this need, it is important to choose an appropriate donor site and to cover the defect completely [1].

As a donor site, the peroneal flap was introduced by Taylor et al. [2] in 1975. It was mainly used for grafting the vascularized fibula, and a cutaneous flap served only as a skin paddle or a buoy for the blood circulation. Peroneal flap surgery remains an important method of vascularized bone grafting. The cutaneous 
flap was introduced by Yoshimura et al. [3,4] in the 1980s, and many other types of flaps, including island and free flaps, have been used since then.

We have applied peroneal flaps to many different sites. We have used island flaps for reconstructing a lower limb around the donor site, retrograde flaps for reconstructing the lower third of a lower limb and an ankle, and anterograde flaps for reconstructing the upper third of a lower limb and a knee. We have used free flaps for upper-limb reconstruction, including a hand, as well as lower-limb reconstruction, and found them to be particularly useful in reconstructing a defect caused by the removal of head and neck carcinoma.

By applying several types of peroneal flaps to clinical cases, we were able to determine their definite advantages and disadvantages and, based on this information, to present some indications for their use. To use peroneal flaps flexibly, it is essential to have the appropriate anatomical knowledge, particularly about the peroneal artery and its perforators. We obtained the required anatomical information by examining cadavers. This paper reports the results of a cadaveric study and clinical research, along with a literature review.

\section{METHODS}

\section{Anatomical study}

We used 2 fresh female cadavers to conduct an anatomical study of the peroneal artery and its perforators in the lower limbs. The pedicle was carefully anatomized under a $\times 3.5$ loupe. The skin incision was performed from the fibula head to the lateral malleolus on the lateral side of one of the lower limbs. Fasciotomy was used for identifying perforators, along which dissection was carried out in the proximal direction. The lower limb was horizontally trisected to determine the distribution and number of perforators. The length of a perforator, which was defined the distance that it traveled from its origin in the major vessel in the lower limb to the point from which it came through the fascia, was measured. Perforators in the lower limbs are generally divided into two types: septocutaneous perforators, which come out vertically along the fascia, and musculocutaneous perforators, which travel intramuscularly to reach the skin. We examined the path traveled by each perforator and the distribution of the perforators in each location. We also determined the major vessel in the lower limb from which the perforator originated (Fig. 1).

\section{Clinical study}

We conducted retrospective research based on the records of 29 patients in whom a peroneal flap had been used for reconstruct-

\section{Fig. 1. Cadaveric dissection of middle third of right leg}

Three perforators arise from the peroneal vessel. The upper one is a musculocutaneous perforator, and the others are septocutaneous (on the lateral surface). M, musculocutaneous; SC, septocutaneous.



ing a defect between February 2005 and June 2012. Follow-up visits were attended by all patients, and the mean follow-up period was 154.8 days (range, 36-494 days). The medical records and clinical photographs for each patient were reviewed to determine the patient's age and gender, the cause of the defect, the defect site and size, surgery type, methods of suturing the donor site, and postoperative complications (Table 1).

\section{Surgical methods}

The fibula head and the lateral malleolus were marked on the lateral side of a lower limb, and an imaginary line for the lateral margin of the fibula was drawn. Along the line, we located a perforator through a handheld Doppler probe. We designed a flap to fit the size of the defect around the location of the perforator as a pedicle. A tourniquet was tightened to prevent bleeding, and a skin incision was made along the posterior interface of the flap. Subfascial dissection was used for inducing the anterior elevation of the flap, and the perforators distributing to the skin through the fascia were exposed. We incised the other anterior interface of the flap and reached the perforators through the subfascial dissection, from which we chose a reliable perforator with the largest vessel diameter and the strongest pulsation and dissected it in the reverse direction to its origin in a major vessel.

\section{Retrograde island peroneal flap}

This type of flap can be used for reconstructing the lower third of a lower limb and the ankle. We designed a flap containing a perforator and placed it between the upper and the middle third of a lower limb. A peroneal vessel was ligated and isolated approximately $5 \mathrm{~mm}$ upward from the origin of the perforator. The blood circulation of the flap with the retrograde blood flow to the 
Table 1. Clinical features of the patients

\begin{tabular}{|c|c|c|c|c|c|c|c|}
\hline Patient & Age $(y r) / S e x$ & Etiology & Location & Flap size $(\mathrm{cm})$ & $\begin{array}{l}\text { Operation } \\
\text { name }\end{array}$ & $\begin{array}{c}\text { Donor site } \\
\text { (closure method) }\end{array}$ & $\begin{array}{c}\text { Complications/Secondary } \\
\text { procedures }\end{array}$ \\
\hline 1 & 63/Female & Trauma & Knee, Rt. & $4.5 \times 7.0$ & APPF & Skin graft & None \\
\hline 2 & 66/Female & Trauma & Ankle, Lt. & $4.5 \times 7.5$ & RPPF & Primary closure & None \\
\hline 3 & 51/Female & MM & Hand, Rt. & $5.0 \times 5.0$ & FPF & Primary closure & None \\
\hline 4 & 52/Female & $\mathrm{SCC}$ & Pharynx & $4.0 \times 4.5$ & FPF & Primary closure & None \\
\hline 5 & 44/Female & Trauma & Upper third of leg, Lt. & $4.0 \times 6.5$ & APPF & Skin graft & Partial flap necrosis/None \\
\hline 6 & 68/Male & $\mathrm{SCC}$ & Tongue & $4.0 \times 6.0$ & FPF & Primary closure & None \\
\hline 7 & 74/Female & $\mathrm{SCC}$ & Tongue & $4.5 \times 6.5$ & FPF & Primary closure & None \\
\hline 8 & 29/Male & Trauma & Great toe, Rt. & $2.0 \times 4.5$ & FPF & Primary closure & None \\
\hline 9 & 62/Male & Trauma & Upper third of leg, Lt. & $4.5 \times 7.0$ & APPF & Skin graft & Partial flap necrosis/Skin graft \\
\hline 10 & 50/Male & Trauma & Ankle, Lt. & $5.5 \times 8.0$ & RPPF & Skin graft & None \\
\hline 11 & 69/Male & Trauma & Foot dorsum, Rt. & $4.0 \times 6.5$ & FPF & Primary closure & None \\
\hline 12 & 80/Female & Trauma & Lower third of leg, Rt. & $4.5 \times 7.5$ & RPPF & Primary closure & Partial flap necrosis/None \\
\hline 13 & 84/Female & $\mathrm{SCC}$ & Pharynx & $6.0 \times 8.0$ & FPF & Skin graft & None \\
\hline 14 & 51/Female & Trauma & Lower third of leg, Rt. & $4.0 \times 6.5$ & RPPF & Primary closure & None \\
\hline 15 & 52/Female & Trauma & Ankle, Lt. & $4.0 \times 7.0$ & RPPF & Primary closure & Partial flap necrosis/None \\
\hline 16 & 44/Female & Trauma & Lower third of leg, Lt. & $6.0 \times 8.0$ & RPPF & Skin graft & None \\
\hline 17 & 68/Male & SCC & Buccal mucosa & $4.0 \times 4.5$ & FPF & Primary closure & None \\
\hline 18 & 74/Female & Trauma & Ankle, Lt. & $5.0 \times 8.0$ & RPPF & Skin graft & None \\
\hline 19 & 33/Male & Trauma & Wrist, Rt. & $5.5 \times 7.5$ & FPF & Skin graft & None \\
\hline 20 & 62/Male & $\mathrm{SCC}$ & Tongue & $4.5 \times 7.5$ & FPF & Skin graft & None \\
\hline 21 & 50/Male & Trauma & Foot dorsum, Lt. & $6.0 \times 8.0$ & FPF & Skin graft & None \\
\hline 22 & 69/Male & Trauma & Hand, Rt. & $5.0 \times 6.5$ & FPF & Primary closure & None \\
\hline 23 & 80/Female & Trauma & Knee, Rt. & $4.5 \times 5.0$ & APPF & Skin graft & Partial flap necrosis/Skin graft \\
\hline 24 & 84/Female & SCC & Upper third of leg, Rt. & $4.0 \times 8.0$ & APPF & Skin graft & None \\
\hline 25 & 51/Female & Trauma & Lower third of leg, Rt. & $4.5 \times 7.5$ & RPPF & Primary closure & None \\
\hline 26 & 55/Female & Trauma & Ankle, Lt. & $4.0 \times 8.0$ & FPF & Primary closure & None \\
\hline 27 & 44/Female & $\mathrm{MM}$ & Hand, Rt. & $8.0 \times 8.0$ & FPF & Skin graft & None \\
\hline 28 & 50/Male & Trauma & Foot dorsum, Lt. & $6.5 \times 8.0$ & FPF & Skin graft & None \\
\hline 29 & 80/Female & SCC & Tonsil, Rt. & $5.0 \times 5.5$ & FPF & Primary closure & None \\
\hline
\end{tabular}

\section{Fig. 2. Case of retrograde island peroneal flap surgery}

(A) Flap design and preoperative perforator localization determined using a Doppler examination. (B) The flap was completely dissected with the main trunk of the peroneal vessels maintaining a patent retrograde flow of the pedicle. (C) Postoperative photograph (2 months) showing a flap that had survived well without any complications.
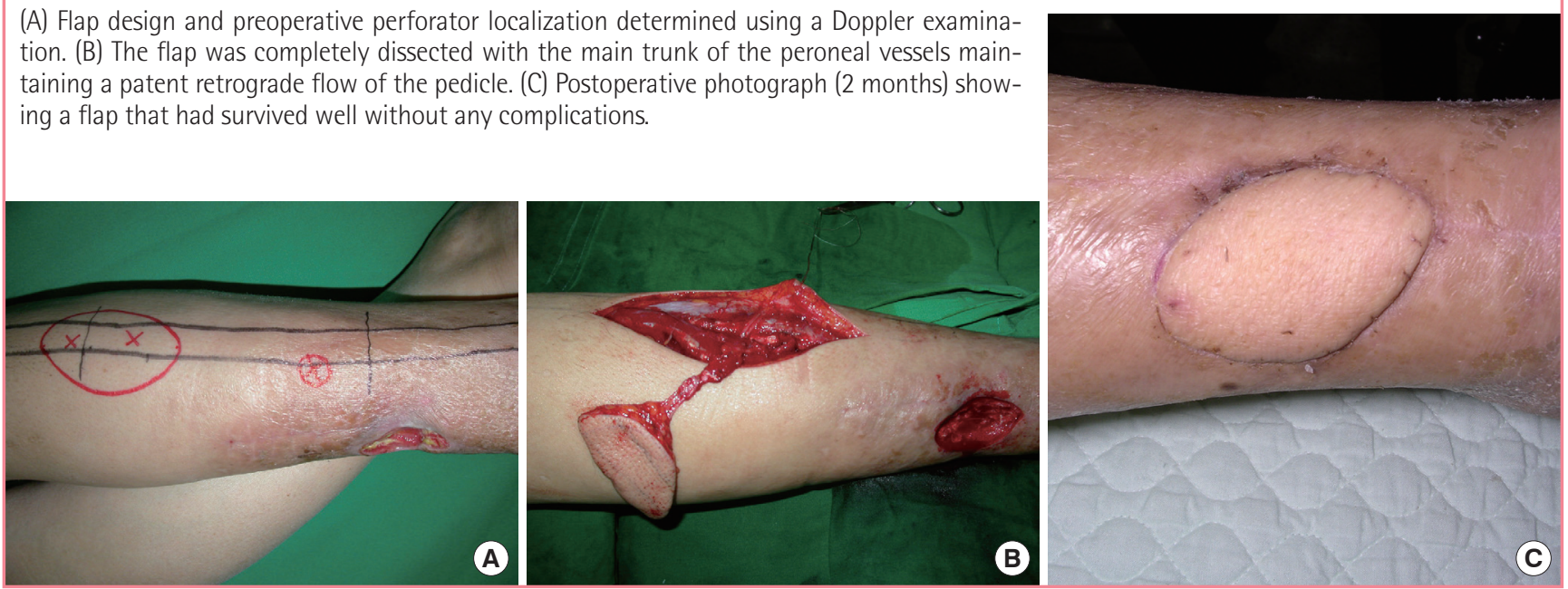

flap was examined, and the peroneal vessel was dissected completely in the lower limb to elevate the flap. The peroneal vessel was dissected up to the pivot point, and the flap was then moved through a subcutaneous tunnel to cover the defect (Fig. 2). 


\section{Fig. 3. Case of anterograde island peroneal flap surgery}

(A) Flap design for the anterograde pedicled peroneal flap. (B) Inset flap and the donor site covered with a skin graft.
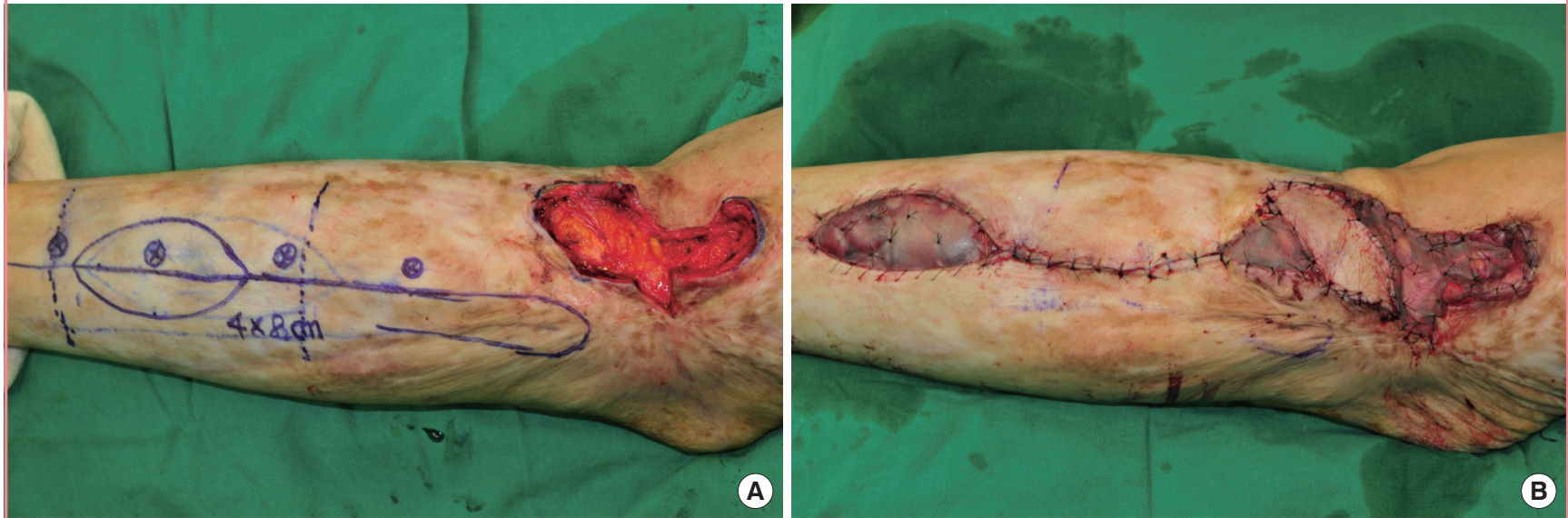

Fig. 4. Peroneal free flap surgery (patient no. 27)

(A) A $7.5 \times 7.5$-cm skin defect after wide excision of malignant melanoma on the right hand. (B) Flap design on the lateral side of the leg for the free peroneal perforator flap. (C) Harvested free peroneal perforator flap including the main trunk of the peroneal vessels for lengthening the pedicle. (D) Immediate postoperative view.
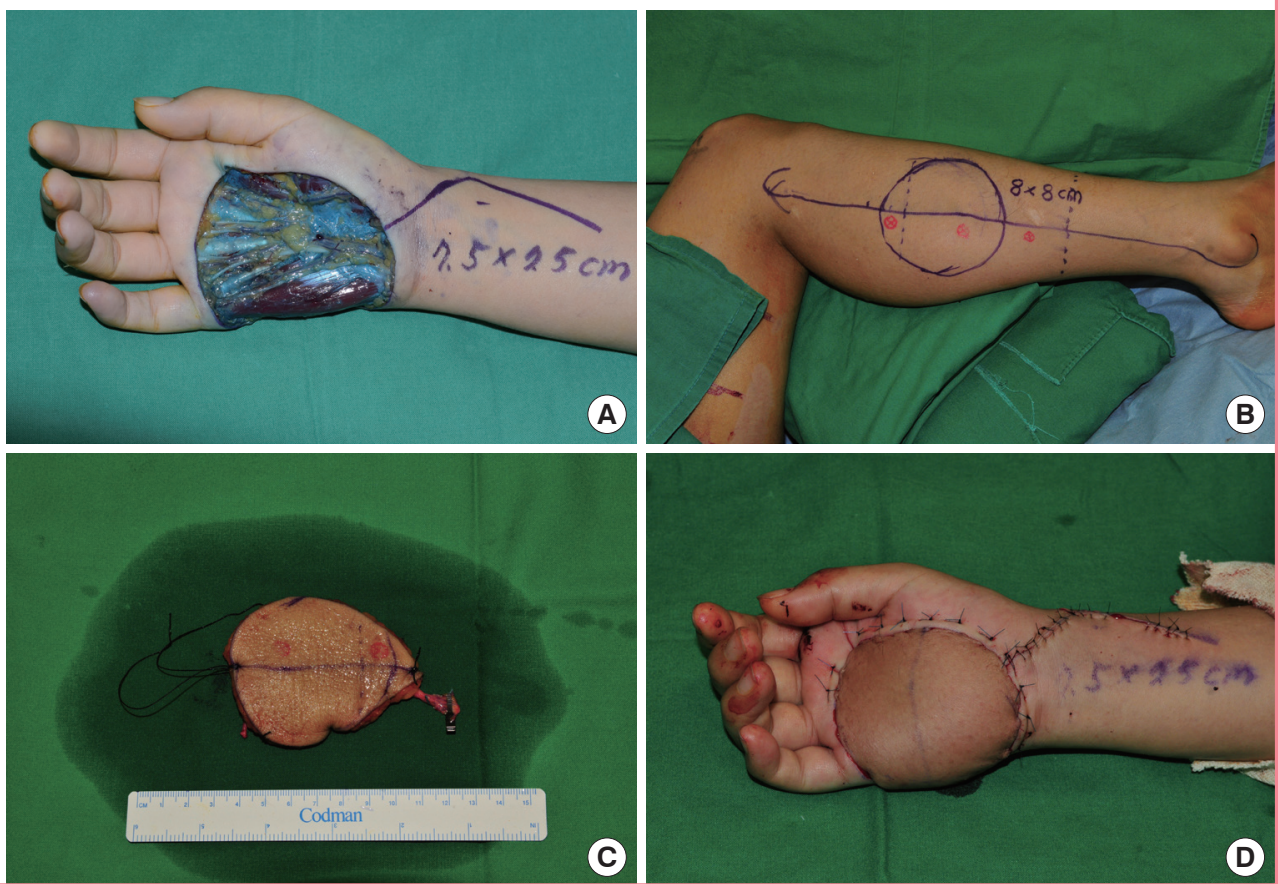

\section{Anterograde island peroneal flap}

This type of flap can be used for reconstructing the upper third of a lower limb and the knee. We designed a flap containing a perforator and placed it between the lower and the middle third of one of the lower limbs. A peroneal vessel was ligated and isolated approximately $5 \mathrm{~mm}$ downward from the origin of the perforator. The peroneal vessel was dissected completely up to the pivot point, and the flap was elevated and moved through a subcutaneous tunnel to cover the defect (Fig. 3).

\section{Free peroneal flap}

We positioned a flap between the upper third and the middle third so that the donor site could be closed directly, if possible. If only a perforator is included as a pedicle, it is possible to elevate a flap without injuring any major vessels. When the perforator was insufficient to cover the necessary length of the pedicle, the major vessels were included. The recipient site was covered with the flap; then, the arteries and venae comitantes of the flap were anastomosed to the vessels at the recipient site (Figs. 4-6).

\section{RESULTS}

\section{Anatomical study}

We found 19 perforators in 4 legs of 2 cadavers, approximately 


\section{Fig. 5. Peroneal free flap surgery (patient no. 21)}

(A) Harvested free peroneal perforator flap including the main trunk of the peroneal vessels for lengthening the pedicle. (B) Postoperative photograph (2 months) showing a flap that had survived well without any complications.
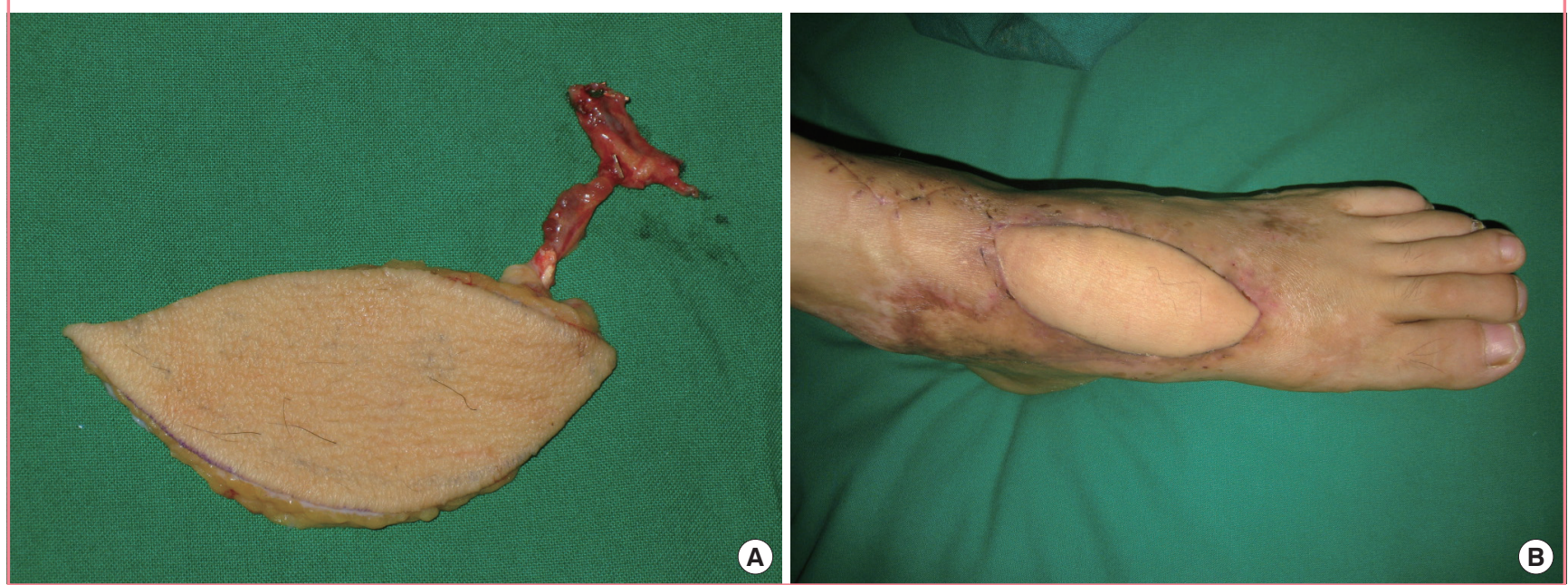

Fig. 6. Peroneal free flap surgery (patient no. 6)

(A) Remaining tongue after hemiglossectomy. (B) A $4 \times 6$-cm flap was harvested without any injury of the major vessels. (C) Immediate postoperative view.
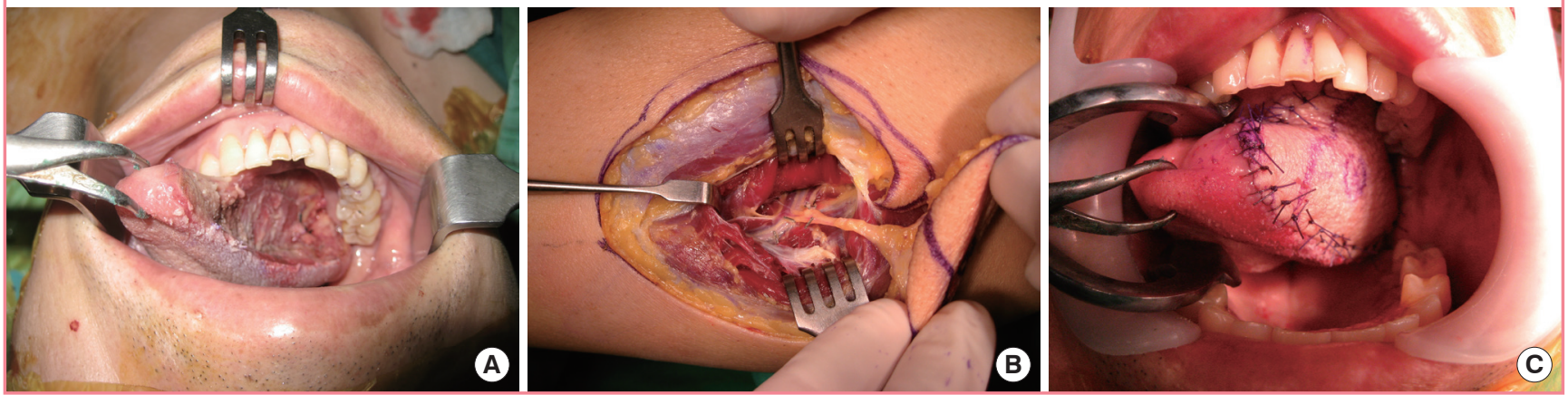

4.8 perforators per leg. Five perforators were found in the upper third of a lower limb, 9 in the middle third, and 5 in the lower third. The length of these perforators ranged from 3.5 to $7.5 \mathrm{~cm}$ (mean, $4.8 \mathrm{~cm}$ ). There were 12 septocutaneous perforators and 7 musculocutaneous perforators. Only musculocutaneous perforators were found in the upper third of a lower limb, and only septocutaneous perforators in the lower third. Three perforators in the upper third and 1 in the middle third originated in the posterior tibial artery; all the others originated in the peroneal artery.

\section{Clinical study}

For this study, we selected 29 patients, 11 males and 18 females. They were aged between 29 years and 84 years (mean, 59.9 years). Ten cases had a defect caused by carcinoma removal, and the remaining 19 had a traumatic defect. Nine cases had a defect in the lower third of a lower limb or an ankle, 5 in the upper third of a lower limb or a knee, 4 in a foot or a toe, 4 in an upper limb or a hand, and 7 in the head and neck. The defects ranged from $2.0 \mathrm{~cm} \times 4.5 \mathrm{~cm}$ to $8.0 \mathrm{~cm} \times 8.0 \mathrm{~cm}$ in size; in 14 cases, the donor site was covered using skin grafting because it could not be closed directly. Retrograde island peroneal flap surgery was conducted in 8 cases, anterograde island peroneal flap surgery in 5 cases, and free peroneal flap surgery in 16 cases. Partial flap necrosis occurred in 5 cases, 2 of which required secondary surgery using skin grafting. No other sequelae occurred (Table 1).

\section{Cases}

Case 1: patient no. 14

A 51-year-old woman developed soft tissue necrosis in the lower third of her right lower limb from a traffic accident. The debridement of necrotic tissues revealed a $6.5 \times 4.0-\mathrm{cm}$ soft tissue defect. The perforators were located using a handheld Doppler probe, and a flap was designed to contain 2 perforators. To per- 
form retrograde island peroneal flap surgery, the peroneal artery and comitant veins were ligated in the upper part of the origin of a perforator as a pedicle in the peroneal artery. The flap was elevated to the pivot point and was moved through a subcutaneous tunnel to the defect in order to cover it. The donor site was closed directly (Fig. 2).

\section{Case 2: patient no. 24}

An 84-year-old woman who had squamous cell carcinoma in a burn scar on the right popliteal fossa underwent wide excision to cover a $2-\mathrm{cm}$ free margin. This procedure caused a deep defect, which covered $4.0 \times 5.0 \mathrm{~cm}$ of the fascia, and repair was arranged for scar contracture. The deep defects caused by skin carcinoma removal were covered with a $4.0 \times 8.0-\mathrm{cm}$ anterograde island peroneal flap, and the defects caused by scar contracture repair and at the donor site were reconstructed using split-thickness skin grafting (Fig. 3).

\section{Case 3: patient no. 27}

A 44-year-old woman underwent wide excision, which covered a $2-\mathrm{cm}$ free margin, to treat malignant melanoma, and was left with a $7.5 \times 7.5-\mathrm{cm}$ defect. An $8.0 \times 8.0-\mathrm{cm}$ flap was elevated to contain 2 perforators from the lateral middle third of the right lower limb. In addition to the perforators, some peroneal vessels were included to obtain a longer pedicle. The peroneal artery and 2 comitant veins in the flap were anastomosed end-to-end to the palmar carpal branch of the radial artery as well as to the comitant and superficial veins at the recipient site; then, splitthickness skin grafting was performed at the donor site (Fig. 4).

\section{Case 4: patient no. 21}

A 50-year-old man developed soft tissue necrosis on the dorsum of the left foot from a traffic accident. A $6.0 \times 8.0-\mathrm{cm}$ flap was elevated from the lateral part of the right lower limb to fit the size of the defect. In addition to the perforators, some peroneal vessels were included to obtain a longer pedicle. The perforator and 2 of its comitant veins in the flap were anastomosed end-toend to the anterior tibial artery as well as to the comitant and superficial veins at the recipient site; split thickness skin grafting was performed at the donor site (Fig. 5).

\section{Case 5: patient no. 6}

In a 68-year-old man who had half of his tongue removed because of tongue cancer, a $4.0 \times 6.0-\mathrm{cm}$ flap was elevated from the lateral part of the left lower limb to contain only perforators. The perforators and their comitant veins in the flap were anastomosed end-to-end to the lingual artery and its comitant veins at the recipient site. The donor site was closed directly (Fig. 6).

\section{DISCUSSION}

Of the blood vessels primarily responsible for the blood supply in the lower limbs, the peroneal artery is one of the best areas to elevate a perforator flap not only because it serves as a feeding artery in the fibula but also because it supplies blood to the skin in the lateral part of the lower limb through a number of musculocutaneous and septocutaneous perforators originating in the fibula $[5,6]$. The peroneal artery originating in the posterior tibia artery travels downward along the posterointerior side of the fibula and generates several perforators to supply blood to the fibula, neighboring muscles, and the skin on the lateral surface along the travel path. Thus, it supplies blood to the area ranging from the middle third to the lower third of the lateral side of the lower limb [7]. Musculocutaneous perforators, which supply the skin with blood through the peroneus longus or soleus, are distributed from the upper distal third to the middle third. Septocutaneous perforators, which supply blood directly to the skin through the septum between the peroneus longus and soleus, are distributed from the middle third to the lower third $[3,8]$. In our cadaveric study, only musculocutaneous perforators were found in the upper part, only septocutaneous perforators in the lower part, and both types of perforators in the middle part.

The lateral part of the lower limb usually contains 3 to 8 perforators $[5,9]$, most of which originate in the peroneal artery, whereas proximal perforators do not always originate in the peroneal artery [10]. In our cadaveric study, 4 of the 19 perforators were musculocutaneous perforators, which originated in the posterior tibial artery; 3 of them were distributed in the upper third, and 1 in the middle third. Retrograde island peroneal flap surgery can be performed only when the major vessel used as a pedicle is the peroneal artery. It is important to note that some perforators, particularly musculocutaneous perforators, in the upper and middle parts may not originate in the peroneal artery.

The perforators in the peroneal artery average about $5 \mathrm{~cm}$ in length, although this measurement has been reported to vary in the literature [11]. In our cadaveric study, the length ranged from 3.5 to $7.5 \mathrm{~cm}$ (mean, $4.8 \mathrm{~cm}$ ). The perforators in the lower third were shorter than those in the middle and upper parts, and musculocutaneous perforators tended to be longer because of their complicated travel path. It is therefore desirable to design a flap in the middle and upper parts with the aim of securing the length of the pedicle during free flap surgery.

Partial flap necrosis occurred as a complication in 5 cases. A problem related to the blood circulation was found in 3 cases of anterograde island peroneal flap surgery and in 2 cases of retrograde island peroneal flap surgery (5 of 16). In free flap surgery, however, no partial flap necrosis occurred ( 0 of 13 ). Thus, an is- 
land flap is at a higher risk of causing a problem related to the blood circulation than a free flap. This is probably because an island flap, which needs a long pedicle to travel up to the defect through a subcutaneous tunnel, can develop inhibited blood circulation due to postoperative edema. In the case of trauma, it may be difficult to elevate a flap from the same trauma site.

It is most desirable to close a donor site directly, taking aesthetics into account. However, the possibility of direct closure varies by donor site location and according to the room, elasticity, and resilience of the skin, all of which are affected by the patient's age. In the lower third of a lower limb, the flaps ranged from 4.0 to $4.5 \mathrm{~cm}$ in diameter, and each case required skin grafting. In the upper and middle third of a lower limb, direct closure was possible when a flap was $<4.5 \mathrm{~cm}$ in diameter, and even when it was $5 \mathrm{~cm}$ in diameter in some cases.

Peroneal flaps have many advantages, none of which are found in other types of flaps. They are very thin and flexible, and require no additional surgery for thinning; therefore, they are very effective in the reconstruction of the head and neck, particularly in the partial reconstruction of the tongue and in hand or foot reconstruction. The donor site can be closed directly, taking into account the flap size, patient age, and so on, and minimum donor site morbidity occurs. A peroneal flap can also be used as a composite flap, which contains bones, muscles, and nerves. A free flap makes it easy to perform dissection up to a major vessel and causes no injury to the major vessels in a lower limb. Another major advantage is the possibility of tightening a tourniquet during surgery.

These techniques have several disadvantages. With the continuing increase of diabetes and aging in the population, it is essential to preserve the major vessels of lower limbs. Injuring the peroneal artery, one of the major vessels in a lower limb, however, is unavoidable when a pedicled flap is used. Therefore, we considered other methods, such as true perforator flaps, prior to major vessel sacrifice. If such methods were impossible, we used a peroneal flap. Failed direct closure of the donor site, which is exposed, can produce very unsatisfactory results aesthetically. The patient needs to be in a prone position, or at least in a lateral position, to expose the peroneal artery, which is medial to the fibula. As a result, it is necessary to change the patient's position, and the location of the vessel makes it difficult to dissect intraoperatively. When a retrograde island peroneal flap is selected, it is necessary to determine the major vessel from which the perforator originates. Our anatomic study showed that some perforators originated in the posterior tibial artery. This uncertainty causes major difficulties, and makes it necessary to select another type of surgery when the perforator originates in any major artery other than the peroneal artery. Furthermore, when varia- tions and malformations of the vessels are present, a preoperative evaluation using angiography is recommended $[12,13]$.

If the surgical method and indications are properly determined to overcome the limitations of the clinical application of island flaps and the disadvantages of a donor site on the basis of a good anatomical understanding of the peroneal artery and its perforators, peroneal flap surgery can be considered a good method to reconstruct diverse sites, resulting in a lower morbidity rate at the donor site.

\section{REFERENCES}

1. Morris SF, Tang M, Almutari K, et al. The anatomic basis of perforator flaps. Clin Plast Surg 2010;37:553-70.

2. Taylor GI, Miller GD, Ham FJ. The free vascularized bone graft: a clinical extension of microvascular techniques. Plast Reconstr Surg 1975;55:533-44.

3. Yoshimura M, Imura S, Shimamura K, et al. Peroneal flap for reconstruction in the extremity: preliminary report. Plast Reconstr Surg 1984;74:402-9.

4. Yoshimura M, Shimada T, Imura S, et al. Peroneal island flap for skin defects in the lower extremity.J Bone Joint Surg Am 1985;67:935-41.

5. Chen YL, Zheng BG, Zhu JM, et al. Microsurgical anatomy of the lateral skin flap of the leg. Ann Plast Surg 1985;15: 313-8.

6. Schaverien M, Saint-Cyr M. Perforators of the lower leg: analysis of perforator locations and clinical application for pedicled perforator flaps. Plast Reconstr Surg 2008;122: 161-70.

7. Yoshimura M, Shimada T, Hosokawa M. The vasculature of the peroneal tissue transfer. Plast Reconstr Surg 1990;85: 917-21.

8. Heitmann C, Khan FN, Levin LS. Vasculature of the peroneal artery: an anatomic study focused on the perforator vessels. J Reconstr Microsurg 2003;19:157-62.

9. Beppu M, Hanel DP, Johnston GH, et al. The osteocutaneous fibula flap: an anatomic study. J Reconstr Microsurg 1992;8:215-23.

10. Yajima H, Ishida H, Tamai S. Proximal lateral leg flap transfer utilizing major nutrient vessels to the soleus muscle. Plast Reconstr Surg 1994;93:1442-8.

11. Papadimas D, Paraskeuopoulos T, Anagnostopoulou S. Cutaneous perforators of the peroneal artery: cadaveric study with implications in the design of the osteocutaneous free fibular flap. Clin Anat 2009;22:826-33.

12. Lee JS, Patel KM, Zou Z, et al. Computerized tomographic and magnetic resonance angiography for perforator-based 
free flaps: technical considerations. Clin Plast Surg 2011;38: 219-28.

13. Alonso-Burgos A, Garcia-Tutor E, Bastarrika G, et al. Preoperative planning of deep inferior epigastric artery perforator flap reconstruction with multislice-CT angiography: imaging findings and initial experience. J Plast Reconstr Aesthet Surg 2006;59:585-93. 